\title{
PEDS: Passivity Enforcement for Descriptor Systems via Hamiltonian-Symplectic Matrix Pencil Perturbation
}

\author{
Yuanzhe Wang ${ }^{1}$, Zheng Zhang ${ }^{1}$ \\ ${ }^{1}$ Department of Electrical and Electronic Engineering \\ The University of Hong Kong, Pokfulam Road, Hong Kong \\ Email: \{yzwang,zzhang,gpang,nwong\}@eee.hku.hk
}

\author{
Cheng-Kok Koh ${ }^{2}$, Grantham K. H. Pang ${ }^{1}$, Ngai Wong ${ }^{1}$ \\ ${ }^{2}$ School of Electrical and Computer Engineering \\ Purdue University, West Lafayette, Indiana \\ Email: chengkok@purdue.edu
}

\begin{abstract}
Passivity is a crucial property of macromodels to guarantee stable global (interconnected) simulation. However, weakly nonpassive models may be generated for passive circuits and systems in various contexts, such as data fitting, model order reduction (MOR) and electromagnetic (EM) macromodeling. Therefore, a post-processing passivity enforcement algorithm is desired. Most existing algorithms are designed to handle poleresidue models. The few algorithms for state space models only handle regular systems (RSs) with a nonsingular $D+D^{T}$ term. To the authors' best knowledge, no algorithm has been proposed to enforce passivity for more general descriptor systems (DSs) and state space models with singular $D+D^{T}$ terms. In this paper, a new post-processing passivity enforcement algorithm based on perturbation of Hamiltonian-symplectic matrix pencil, PEDS, is proposed. PEDS, for the first time, can enforce passivity for DSs. It can also handle all kinds of state space models (both RSs and DSs) with singular $D+D^{T}$ terms. Moreover, a criterion to control the error of perturbation is devised, with which the optimal passive models with the best accuracy can be obtained. Numerical examples then verify that PEDS is efficient, robust and relatively cheap for passivity enforcement of DSs with mild passivity violations.
\end{abstract}

\section{INTRODUCTION}

Passivity is a crucial property in the macromodeling of circuits and systems, as a stable but nonpassive system may produce an unstable global system when interfaced with other systems [10]. Roughly speaking, a system is regarded as passive if it is incapable of generating energy. Note that passivity is an input-output property of a system and is independent of the internal realization. Precise definition of passivity involves positive realness (for admittance/impedance representation) or bounded realness (for S-parameter representation) of transfer functions. Due to the importance of passivity in global simulation, many theories addressing passivity check [1], [5], [13], [23], [24] and passivity enforcement [6], [7], [18] have been proposed.

Nonpassive models may be generated from passive systems in various contexts, such as model order reduction (MOR), data fitting, electromagnetic (EM) modeling etc. due to computation inaccuracy or other reasons. In the context of MOR, reduced-order models of passive original models may become nonpassive [4], [12]. PRIMA can preserve passivity only for definite state space structures (which constitute only a small subclass of passive state space models) [15]. Positive-real balanced truncation is not applicable to large initial models and may be inaccurate for descriptor systems (DSs) [16]. In the context of data fitting, nonpassive models of passive underlying systems may be generated due to sampling and/or fitting error, such as the cases of vector fitting (VF) and Loewner matrix-based tangential interpolation (LoewnerTI) [8], [11], [21]. To the authors' best knowledge, there is no efficient, general-purpose Loewner-TI that produces passivityguaranteed models. In the context of EM modeling, discretization procedure may result in nonpassive models for passive structures [17]. In all these cases, passivity violations are usually small and perturbation-based post-processing passivity enforcement is possible.

Most existing passivity enforcement algorithms only handle pole-residue models, which arise naturally from VF. However, in most cases the nonpassive models to be dealt with are in the state space form, such as nonpassive models generated by MOR, EM modeling and Loewner-TI. The few passivity enforcement algorithms designed for state space models are restricted to regular systems (RSs) and rely on the assumption that $D+D^{T}$ is nonsingular [6], [18]. Nevertheless, circuits and systems are naturally described as DSs instead of RSs [3]. Moreover, $D+D^{T}$ may be singular or even zero for a large number of models such as modified nodal analysis (MNA) models of RCL circuits [9] and models generated by LoewnerTI. Therefore, the new algorithm PEDS is proposed in this paper. Tables I and II illustrate the model types generated by various modeling techniques and the application scopes of the different passivity enforcement algorithms.

The framework of PEDS is as follows. For an impulse-free DS, passivity can be enforced by perturbing the generalized eigenvalues of the Hamiltonian-symplectic matrix pencil. For a DS with an improper part, the improper part can be extracted through an efficient algorithm which only involves matrixvector multiplication and matrix-vector solver. The improper part can then be compensated through a linear matrix inequality (LMI) method and the proper part can be perturbed as an impulse-free DS. For a model (either RS or DS) with singular 
TABLE I

MODEL TYPES GENERATED BY DIFFERENT MODELING TECHNIQUES

\begin{tabular}{c|c|c|c|c}
\hline \hline modeling techniques & pol-res & RS-non & RS-sin & DS \\
\hline VF & $\sqrt{ }$ & & & \\
\hline Loewner-TI & & & & $\sqrt{ }$ \\
\hline MOR & & $\sqrt{ }$ & $\sqrt{ }$ & $\sqrt{ }$ \\
\hline EM & & $\sqrt{ }$ & $\sqrt{ }$ & $\sqrt{ }$ \\
\hline \hline
\end{tabular}

${ }^{1}$ Pol-res represents pole-residue model; RS-non represents RS with nonsingular $D+D^{T}$; RS-sin represents RS with singular $D+D^{T}$. Same abbreviations are used in Table II

TABLE II

APPLICATION SCOPES OF DIFFERENT PASSIVITY ENFORCEMENT ALGORITHMS

\begin{tabular}{c|c|c|c|c}
\hline \hline enforcement algorithms & pol-res & RS-non & RS-sin & DS \\
\hline pole-residue perturbation & $\sqrt{ }$ & & & \\
\hline Hamiltonian perturbation [6] & $\sqrt{ }$ & $\sqrt{ }$ & & \\
\hline proposed PEDS & $\sqrt{ }$ & $\sqrt{ }$ & $\sqrt{ }$ & $\sqrt{ }$ \\
\hline \hline
\end{tabular}

$D+D^{T}$, a model conversion is first performed. The resulting model is an impulse-free DS if the original model is an RS, and a DS with the same index if the original model is a DS. The perturbations of the proper and the improper part (if any) are finally converted to a standard least-square problem and a standard "mincx" problem, the solutions of which are trival and efficient. Optimal perturbations with best accuracy are guaranteed through solving the two standard problems.

The main contributions of the paper are: (i)The proposed PEDS is the first algorithm that can enforce passivity for DSs, and thus has a much wider application scope. (ii)PEDS is able to handle systems with singular $D+D^{T}$ through a model conversion. (iii)A criterion to control the error of perturbation and a method to obtain optimal passive models are developed. (iv)A cheap and efficient algorithm for extraction and optimal perturbation of the improper part of a DS is introduced. Numerical examples have verified that PEDS is efficient, robust and relatively cheap for passivity enforcement of DSs with small passivity violations.

The remainder of the paper is as follows. Section II introduces the background knowledge. Section III introduces the system decomposition and passivity violation characterization methods. PEDS is proposed in Section IV. Section V gives numerical examples to verify the proposed algorithm and Section VI draws the conclusion.

\section{BACKGROUND}

\section{A. Descriptor Systems}

Many linear time-invariant circuits and systems can be naturally described by a DS [3]:

$$
\begin{gathered}
E \dot{x}(t)=A x(t)+B u(t), \\
y(t)=C x(t)+D u(t),
\end{gathered}
$$

where $x(t) \in \mathbb{R}^{n}, u(t), y(t) \in \mathbb{R}^{m}, E, A \in \mathbb{R}^{n \times n}, B \in$ $\mathbb{R}^{n \times m}, C \in \mathbb{R}^{m \times n}, D \in \mathbb{R}^{m \times m}$. $E$ is generally singular, otherwise (1) can be reduced to an RS. The matrix pencil $(A, E)$ is assumed to be regular, i.e., there exists at least one $s_{0}$ such that $\left(s_{0} E-A\right)$ is nonsingular. The transfer function of (1) is

$$
H(s)=C(s E-A)^{-1} B+D .
$$

Under the regular matrix pencil assumption, the system can be rewritten in the Weierstrass canonical form, i.e., there exist nonsingular matrices $W, T$ such that

$$
E=W\left[\begin{array}{cc}
I_{n_{f}} & 0 \\
0 & N
\end{array}\right] T, \quad A=W\left[\begin{array}{cc}
J & 0 \\
0 & I_{n_{\infty}}
\end{array}\right] T,
$$

where $I_{x}$ represents the identity matrix of dimension $x, n_{f}+$ $n_{\infty}=n, N$ is a nilpotent matrix of index $\mu$ (i.e. $N^{\mu-1} \neq 0$ and $N^{\mu}=0$ ). $\mu$ is also called the index of the DS. Thus (2) can be decomposed into the proper part $\left(H_{p}(s)\right)$ and improper part $\left(H_{\infty}(s)\right)$.

$$
H(s)=\underbrace{C_{p}\left(s I_{n_{f}}-J\right)^{-1} B_{p}+M_{0}}_{H_{p}(s)}+\underbrace{\sum_{i=1}^{\mu-1} s^{i} M_{i}}_{H_{\infty}(s)},
$$

where $M_{0}=D-C_{\infty} B_{\infty}, M_{i}=-C_{\infty} N^{i} B_{\infty}$ for $i=$ $1,2 \ldots, \mu-1,\left[\begin{array}{c}B_{p} \\ B_{\infty}\end{array}\right]=W^{-1} B,\left[\begin{array}{cc}C_{p} & C_{\infty}\end{array}\right]=C T^{-1}$.

Besides, it is also beneficial to define the right (left) spectral projector $P_{r}\left(P_{l}\right)$ [cf.(3)], which projects onto the right (left) deflating subspace associated with the finite eigenvalues of $(A, E)$, as [19]

$$
P_{r}=T^{-1}\left[\begin{array}{cc}
I_{n_{f}} & 0 \\
0 & 0
\end{array}\right] T\left(\mathrm{P}_{l}=W\left[\begin{array}{cc}
I_{n_{f}} & 0 \\
0 & 0
\end{array}\right] W^{-1}\right) .
$$

In this paper, we use $\bar{\sigma}$ to represent complex conjugate, ${ }^{T}$ to represent transpose and ${ }^{*}$ to represent (complex) conjugate transpose.

\section{B. Passivity Conditions of a DS}

Passivity can be roughly interpreted as the inability to generate energy. The precise definition of passivity involves positive realness (for admittance/impedance representation) or bounded realness (for S-parameter representation) of the transfer function. Only admittance/impedance representation is considered henceforth, as all the results for admittance/impedance representation can be straightforwardly extended to S-parameter representation. A rational transfer function $H(s)$ is called positive-real if [5]:

(1) $H(s)$ has no poles with positive real parts;

(2) $G(j \omega)=\frac{1}{2}\left(H(j \omega)+H^{*}(j \omega)\right) \geq 0$ for any $j \omega$ that is not a pole of $H(s), \omega \in \mathbb{R}$;

(3) if $j \omega$ or $\infty$ is a pole of $H(s)$, then it is a simple pole and the relevant residue matrix is positive semidefinite.

For a DS (in the admittance/impedance representation) with a transfer function in the decomposition form (4), it is passive if and only if:

(1) the proper part $H_{p}(s)$ is positive real.

(2) the improper part satisfies $M_{1} \geq 0$ and $M_{i}=0$ for $i \geq$ 2 .

Denote $M_{\nu-1}$ as the highest order moment that is not zero (i.e. $M_{\nu-1} \neq 0$ and $M_{\nu}=0$ ). If the $\mathrm{DS}$ is in its minimal 
realization (i.e. the DS is both controllable and observable), $\nu=\mu$, otherwise, $\nu \leq \mu$.

\section{Perturbation of Generalized Eigenvalues}

Consider $\mathcal{J}, \mathcal{K} \in \mathbb{R}^{n \times n}, \lambda \in \mathbb{C}$ is called the generalized eigenvalue of the matrix pencil $(\mathcal{J}, \mathcal{K})$ if there exist solutions $x, y\left(x \neq 0^{n}, y \neq 0^{n}\right)$ of the equations

$$
\mathcal{J} x=\lambda \mathcal{K} x \quad \text { and } \quad y^{*} \mathcal{J}=\lambda y^{*} \mathcal{K}
$$

where $x, y \in \mathbb{C}^{n}$ are called right and left eigenvectors associated with $\lambda$, respectively.

$\lambda$ is conveniently represented by a tuple $\langle\alpha, \beta\rangle\left(|\alpha|^{2}+|\beta|^{2} \neq\right.$ $0)$ which satisfies $\lambda=\alpha / \beta$. If $\beta=0, \lambda$ is an infinite eigenvalue of $(\mathcal{J}, \mathcal{K})$. The tuple, right and left eigenvectors associated with an eigenvalue of $(\mathcal{J}, \mathcal{K})$ can be written together as a generalized eigentriplet $(y,\langle\alpha, \beta\rangle, x)$. If $(\mathcal{J}, \mathcal{K})$ is perturbed by a small matrix pair $(\Delta \mathcal{J}, \Delta \mathcal{K})$, the generalized eigenvalues $\langle\alpha, \beta\rangle=\left\langle y^{*} \mathcal{J} x, y^{*} \mathcal{K} x\right\rangle$ change to

$$
\left\langle\alpha^{\prime}, \beta^{\prime}\right\rangle=\langle\alpha, \beta\rangle+\left\langle y^{*} \Delta \mathcal{J} x, y^{*} \Delta \mathcal{K} x\right\rangle+O\left(\epsilon^{2}\right),
$$

where $\epsilon=\|[\Delta \mathcal{J} \Delta \mathcal{K}]\|_{2}, x, y$ are normalized eigenvectors.

\section{Hamiltonian and Symplectic Matrices}

We also need the definition of Hamiltonian and symplectic matrices. $X \in \mathbb{R}^{2 n \times 2 n}$ is called a Hamiltonian matrix if

$$
J_{0}^{-1} X J_{0}=-X^{T}
$$

where $J_{0}=\left[\begin{array}{cc}0 & I_{n} \\ -I_{n} & 0\end{array}\right]$ satisfies $J_{0}^{T}=J_{0}^{-1}=-J_{0}$. On the contrary, $X \in \mathbb{R}^{2 n \times 2 n}$ is called a symplectic matrix if

$$
J_{0}^{-1} X J_{0}=X^{T} \text {. }
$$

If $\mathcal{J} \in \mathbb{R}^{2 n \times 2 n}$ is Hamiltonian and $\mathcal{K} \in \mathbb{R}^{2 n \times 2 n}$ is symplectic, the generalized eigenvalues $\lambda_{i}$ of the matrix pencil $(\mathcal{J}, \mathcal{K})$ distribute symmetrically on the complex plane w.r.t. both real and imaginary axes. Here is a brief proof.

Proof: It is obvious that if $\mathcal{J}, \mathcal{K}$ are real, the eigenvalues are in conjugate pairs. On the other hand, if $\lambda$ is a generalized eigenvalue of $(\mathcal{J}, \mathcal{K}), \mathcal{J} x=\lambda \mathcal{K} x, x^{T} \mathcal{J}^{T}=\lambda x^{T} \mathcal{K}^{T}$, $-x^{T} J_{0}^{-1} \mathcal{J} J_{0}=\lambda x^{T} J_{0}^{-1} \mathcal{K} J_{0}$. Assume $y^{*}=x^{T} J_{0}^{-1}$, we have $y^{*} \mathcal{J}=-\lambda y^{*} \mathcal{K}$, which means $-\lambda$ is also an eigenvalue of $(\mathcal{J}, \mathcal{K})$. So every $\lambda$ implies coexistence of the tuple $(\lambda, \bar{\lambda},-\lambda,-\bar{\lambda})$.

\section{System DECOMPOSITION AND PASSIVITY IDENTIFICATION}

\section{A. System Decomposition}

According to Section II-B, the passivity characterization and enforcement of DSs involve manipulation of both proper and improper parts, thus a system decomposition should be performed in advance. Here we would like to emphasize that most DSs of interest are in fact impulse-free, thus the system decomposition procedure can be avoided. For DSs with impulse responses, directly computing the Weierstrass canonical form (3) is known to be prohibitively expensive and ill-conditioned. Here, we introduce a new method to calculate $\nu$ and extract the improper part. Consider the transfer function as (4), we calculate the limit

$$
\Gamma=\lim _{s \rightarrow \infty} \frac{H(s)}{s} .
$$

(1) If $\Gamma=0$ (which is the case for most DSs of interest), the DS is impulse-free $(\nu=1)$.

(2) If $\Gamma=\infty$, the system is definitely nonpassive $(\nu>2)$.

(3) If $\Gamma=$ constant $\neq 0, \nu=2$ and $M_{1}=\Gamma$. Thus the improper part $s M_{1}$ has been extracted.

In practice, $\Gamma$ can be calculated by assuming $s$ to be two large positive number (e.g. $s_{1}=1 e 30$ and $s_{2}=1 e 40$ ). If $H\left(s_{2}\right) / s_{2}<<H\left(s_{1}\right) / s_{1}, \Gamma=0$; if $H\left(s_{1}\right) / s_{1}=H\left(s_{2}\right) / s_{2}$, $\Gamma=$ constant; otherwise, $\Gamma=\infty$. The numerical stability and high efficiency of this method has been proved by real-world examples of order from hundreds to tens of thousands.

If $\nu=2$, the proper part can be calculated via canonical projector techniques [14], [22], [25]. Right spectral projector $P_{r}$ can be calculated in three steps following [22]. Then the proper part of (1) is extracted as a new DS $\left(E_{p}=E P_{r}, A, B, C, D\right)$ [cf.(3),(5)]. It is straightforward to verify that the new DS is impulse-free and that its transfer function is identical to the proper part of the original transfer function [cf.(4)].

\section{B. Passivity Violation Characterization}

We start this subsection by introducing the generalized Hamiltonian theorem [24].

Theorem 3.1: For a stable, impulse-free DS as (1), if $\lambda$ is not an eigenvalue of $\frac{D+D^{T}}{2}$, then $\lambda$ is an eigenvalue of $G(j \omega)=\frac{1}{2}\left(H(j \omega)+H^{*}(j \omega)\right)$ if and only if $j \omega$ is a generalized eigenvalue of the matrix pencil $(\mathcal{J}, \mathcal{K})$, where

$$
\begin{aligned}
& \mathcal{J}=\left[\begin{array}{cc}
A+B Q^{-1} C & B Q^{-1} B^{T} \\
-C^{T} Q^{-1} C & -A^{T}-C^{T} Q^{-1} B^{T}
\end{array}\right], \\
& \mathcal{K}=\operatorname{blkdiag}\left(E, E^{T}\right), \quad Q=\left(2 \lambda I-D-D^{T}\right) .
\end{aligned}
$$

We refer the reader to [24] for the proof of this theorem. Here, we would like to point out that it is straightforward to verify that $\mathcal{J}$ is Hamiltonian and $\mathcal{K}$ is symplectic. Hence the spectrum of the matrix pencil $(\mathcal{J}, \mathcal{K})$ is symmetric w.r.t. both real and imaginary axis (cf. Section II-D), which is the basis of the perturbation theory to be introduced.

Proposition 3.2: Assume that the set $\Lambda=\left\{j \omega_{i}\right\} \quad(i=$ $1,2 \ldots, k)$ contains all the purely imaginary generalized eigenvalues of $(\mathcal{J}, \mathcal{K})$ with positive imaginary parts, sorted in ascending order, which divide the frequency band $[0,+\infty)$ into $\mathrm{k}+1$ segments. Then, $G(j \omega)$ at the center frequency of each segment (for the $(\mathrm{k}+1)$ th segment the frequency is selected as $\left.\frac{3}{2} j \omega_{k}\right)$ is computed. If $G(j \omega) \geq 0$ for the segment $\left(j \omega_{i}, j \omega_{i+1}\right)$, then the system is passive in this frequency segment. Otherwise, it is nonpassive in this $\left(j \omega_{i}, j \omega_{i+1}\right)$ frequency segment.

The above proposition provides us with a convenient approach to check the passivity of the DS and pinpoint the frequency bands where passivity violations occur. Note that only generalized eigenvalues with positive imaginary parts are chosen due to the symmetry of the spectrum (cf. Section 
II-D). Compared with the Hamiltonian method for RSs [6], the proposed method does not require the relatively expensive calculation of slopes. With passivity violation information obtained from proposition 3.2, passivity enforcement can then be performed.

\section{PASSIVITY ENFORCEMENT}

\section{A. Passivity Enforcement of Improper Part}

Passivity of a DS requires that $M_{i}=0$ for $i \geq 2$ and $M_{1} \geq 0$. Hence $M_{i}(i \geq 2)$ should be discarded if $\nu>$ 2. To enforce $M_{1}$ to be positive semidefinite, the following optimization problem should be solved

$$
\min _{\tilde{M}_{1}}\left\|\tilde{M}_{1}-M_{1}\right\|_{F} \quad \text { subject to } \quad \tilde{M}_{1} \geq 0 .
$$

The optimization problem (12) can be solved using Matlab LMI toolbox by converting it to a standard 'mincx' problem as follows (where $\operatorname{vec}(X)$ is a column vector constructed by stacking all the columns of the matrix $X$ together):

$$
\left.\begin{array}{l}
\min _{t \in \mathbb{R}} t \\
\text { subject to }\left\{\begin{array}{c}
\tilde{M}_{1}>0 \\
{\left[\begin{array}{c}
t \\
\operatorname{vec}\left(\tilde{M}_{1}-M_{1}\right)
\end{array}\right.}
\end{array} \quad\left(\operatorname{vec}\left(\tilde{M}_{1}-M_{1}\right)\right)^{T}\right. \\
I_{m^{2}}
\end{array}\right]>0
$$

Proof: By induction it can be proven that:

$$
\operatorname{det}\left[\begin{array}{cccc}
t & x_{1} & \cdots & x_{n} \\
x_{1} & 1 & & \\
\vdots & & \ddots & \\
x_{n} & & & 1
\end{array}\right]=t-\sum_{i=1}^{n} x_{i}^{2}
$$

Thus

$$
\left[\begin{array}{cccc}
t & x_{1} & \cdots & x_{n} \\
x_{1} & 1 & & \\
\vdots & & \ddots & \\
x_{n} & & & 1
\end{array}\right]>0 \Leftrightarrow\left\{\begin{array}{c}
t>0 \\
t-x_{1}^{2}>0 \\
\vdots \\
t-\sum_{i=1}^{n} x_{i}^{2}>0
\end{array} \Leftrightarrow t-\sum_{i=1}^{n} x_{i}^{2}>0\right.
$$

Therefore, the second constraint in (13) is equivalent to

$$
t>\sum_{i=1}^{m} \sum_{j=1}^{m}\left|\tilde{M}_{1}(i, j)-M_{1}(i, j)\right|^{2}=\left\|\tilde{M}_{1}-M_{1}\right\|_{F}^{2}
$$

Hence (13) is equivalent to finding the minimal Frobenius norm of $\tilde{M}_{1}-M_{1}$ which satisfies $\tilde{M}_{1}>0$.

Note that the size of $M_{1}$ is $m$ (i.e. the number of ports, which is usually small), hence it is cheap to solve the above optimization problem (the size of the second constraint is $\left.m^{2}+1\right)$. The solution $\tilde{M}_{1}$ can be incorporated into the new guaranteed passive DS as discussed in Section IV-D.

\section{B. Passivity Enforcement of Proper Part}

1) Optimal Perturbation Strategy: Section III-B indicates that the passivity of the proper part can be enforced by perturbing the state space matrices, $A, B, C, D, E$ (for an impulse-free DS) or $E_{p}$ (projected matrix for an impulsive DS) such that the purely imaginary eigenvalues of $(\mathcal{J}, \mathcal{K})$ are moved off the imaginary axis. To develop an algorithm of perturbation, two questions arise immediately: (i) which matrices to be perturbed? and (ii) how to control the error of the perturbation?

The following analyses answer the first question. First, $E$ and $A$ should remain unchanged to guarantee the perturbed system to be stable and to preserve the key dynamic properties of the system (pole distribution). Second, as the application of generalized Hamiltonian theorem involves the assumption that $D+D^{T}$ is nonsingular (if singular, a further equivalent conversion should be performed, cf. Section IV-C). Moreover, perturbation of $D$ will introduce inaccuracy at all frequencies. $D$ is also preserved for these reasons. The only choice is to perturb $B$ and/or $C$, which is convenient as the transfer function is a linear function of $C$ and $B$. Here we choose to perturb $C$ only. Similar choice appears in [6].

To following calculation answers the second question. Assume the impulse response (inverse Laplace transform of transfer function) of the proper part $\left(E_{p}(\right.$ or $\left.E), A, B, C, D\right)$ is $h(t)$, the accuracy of the perturbed system can be measured by

$$
\Delta=\int_{0}^{\infty}\|d h(t)\|_{F}^{2} d t=\int_{0}^{\infty} \operatorname{trace}\left(d h(t) d h^{T}(t)\right) d t
$$

As $d h(t)=d C \mathcal{F}(t) B$, where $\mathcal{F}(t)=$ $T^{-1}\left[\begin{array}{cc}e^{J t} & 0 \\ 0 & 0\end{array}\right] W^{-1}$, we have

$$
\Delta=\operatorname{trace}\left(d C \mathcal{G}_{p c} d C^{T}\right)
$$

Here,

$$
\mathcal{G}_{p c}=\int_{0}^{\infty} \mathcal{F}(t) B B^{T} \mathcal{F}^{T}(t) d t
$$

is called the proper controllability Gramian, which can be solved from the projected generalized Lyapunov equations [19]

$$
\begin{gathered}
E \mathcal{G}_{p c} A^{T}+A \mathcal{G}_{p c} E^{T}=-P_{l} B B^{T} P_{l}^{T} \\
\mathcal{G}_{p c}=P_{r} \mathcal{G}_{p c} .
\end{gathered}
$$

Assume that $\mathcal{G}_{p c}=L^{T} L$ (Cholesky factorization), a coordinate transformation is performed

$$
d C_{t}=d C L^{T} .
$$

Thus,

$$
\Delta=\operatorname{trace}\left(d C_{t} d C_{t}^{T}\right)=\left\|d C_{t}\right\|_{F}^{2}=\left\|\operatorname{vec}\left(d C_{t}\right)\right\|_{2}^{2} .
$$

2) Perturbation of Hamiltonian and symplectic Matrix Pencil: If $C$ is perturbed by a small matrix $d C$, the symplectic matrix $\mathcal{K}$ remains unchanged while the Hamiltonian matrix $\mathcal{J}$ is perturbed by $d \mathcal{J}$. We have

$$
d \mathcal{J}=\left[\begin{array}{cc}
B Q_{0}^{-1} d C & 0 \\
-C^{T} Q_{0}^{-1} d C-d C^{T} Q_{0}^{-1} C & -d C^{T} Q_{0}^{-1} B^{T}
\end{array}\right],
$$

where $Q_{0}=-\left(D+D^{T}\right), \lambda$ is assigned $0 . d \mathcal{J}$ is also readily checked to be Hamiltonian. 
When $\mathcal{J}$ is perturbed by $d \mathcal{J}$ and $\mathcal{K}$ remains unchanged, the generalized eigenvalues of $(\mathcal{J}, \mathcal{K})$ change into [cf.(7)]:

$$
\lambda^{\prime}=\frac{\alpha^{\prime}}{\beta}=\frac{\alpha+\Delta \alpha}{\beta}=\lambda_{0}+\frac{y^{*} d \mathcal{J} x}{y^{*} \mathcal{K} x} .
$$

Besides, assume that $x, y$ are the right and left eigenvectors associated with a purely imaginary generalized eigenvalue of $(\mathcal{J}, \mathcal{K})$, then $y=J_{0} x$. Here is the proof.

Proof: If $x$ is the right eigenvector of $(\mathcal{J}, \mathcal{K})$, we have $\mathcal{J} x=\lambda \mathcal{K} x$. Perform conjugate transpose on both sides, we have $x^{*} \mathcal{J}^{T}=-\lambda x^{*} \mathcal{K}^{T}$ ( $\lambda$ is imaginary, $\mathcal{J}, \mathcal{K}$ are real). As $\mathcal{J}$ is Hamiltonian and $\mathcal{K}$ is symplectic, the previous equation leads to $x^{*}\left(-J_{0}^{-1} \mathcal{J} J_{0}\right)=-\lambda x^{*}\left(J_{0}^{-1} \mathcal{K} J_{0}\right)$. Hence $x^{*} J_{0}^{-1} \mathcal{J}=\lambda x^{*} J_{0}^{-1} \mathcal{K}$. According to the definition of left eigenvector, we have $y^{*}=x^{*} J_{0}^{-1}$, i.e., $y=J_{0} x$.

Subsequently, (23) can be rewritten as

$$
\lambda^{\prime}=\lambda_{0}+\frac{x^{*} J_{0} d \mathcal{J} x}{x^{*} J_{0} \mathcal{K} x} .
$$

It is straightforward to verify that $J_{0} d \mathcal{J}$ is real symmetric and $J_{0} \mathcal{K}$ is real and skew symmetric (i.e. $\left(J_{0} \mathcal{K}\right)^{T}=-J_{0} \mathcal{K}$ ), which indicates that $x^{*} J_{0} d \mathcal{J} x$ is real and $x^{*} J_{0} \mathcal{K} x$ is purely imaginary. As a result, $\lambda^{\prime}$ remains purely imaginary if $\lambda_{0}$ is purely imaginary.

Assuming the $i$ th purely imaginary eigenvalue of $(\mathcal{J}, \mathcal{K})$ is $j \omega_{i}$ and it is supposed to be moved to $j \tilde{\omega}_{i}$, we have [cf.(24)]

$$
j \tilde{\omega}_{i}-j \omega_{i}=\frac{x_{i}^{*} J_{0} d \mathcal{J} x_{i}}{x_{i}^{*} J_{0} \mathcal{K} x_{i}} .
$$

Split $x_{i}$ as two vectors of the same dimension $x_{i}=$ $\left[\begin{array}{l}x_{i, 1} \\ x_{i, 2}\end{array}\right]$, the numerator of (25) can be simplified to

$$
x_{i}^{*} J_{0} d \mathcal{J} x_{i}=-2 \operatorname{Re}\left(z_{i}^{*} d C x_{i, 1}\right),
$$

where $z_{i}=Q_{0}^{-1} C x_{i, 1}+Q_{0}^{-1} B^{T} x_{i, 2}$, and the denominator of (25) can be simplified to

$$
x_{i}^{*} J_{0} \mathcal{K} x_{i}=j(-2) \operatorname{Im}\left(x_{i, 2}^{*} E x_{i, 1}\right) .
$$

Therefore (25) turns into

$$
-\boldsymbol{R e}\left(z_{i}^{*} d C_{t} L^{-T} x_{i, 1}\right)=\left(\tilde{\omega}_{i}-\omega_{i}\right) \operatorname{Im}\left(x_{i, 2}^{*} E x_{i, 1}\right) .
$$

The matrix to be solved in (28) is $C_{t}$. Using the property of Kronecker product

$$
Y=B X A^{T} \Longleftrightarrow \operatorname{vec}(Y)=(A \otimes B) \operatorname{vec}(X),
$$

we can rewrite (28) as

$-\boldsymbol{R e}\left(\left(x_{i, 1}^{T} L^{-1}\right) \otimes z_{i}^{*}\right) \times \operatorname{vec}\left(d C_{t}\right)=\left(\tilde{\omega}_{i}-\omega_{i}\right) \operatorname{Im}\left(x_{i, 2}^{*} E x_{i, 1}\right)$.

In (30), the perturbation matrix (under the coordinate transformation of $\left.L^{-1}\right) C_{t}$ is isolated. Denote

$$
\begin{gathered}
m_{i}=-\boldsymbol{R e}\left(\left(x_{i, 1}^{T} L^{-1}\right) \otimes z_{i}^{*}\right) \\
n_{i}=\left(\tilde{\omega}_{i}-\omega_{i}\right) \operatorname{Im}\left(x_{i, 2}^{*} E x_{i, 1}\right) .
\end{gathered}
$$

If $k$ generalized eigenvalues need to be moved, (30) can be incorporated $k$ times as a matrix format. So the problem becomes

$$
\min \left\|\operatorname{vec}\left(d C_{t}\right)\right\|_{2}, \quad \text { subject to } \mathbf{M} \times \operatorname{vec}\left(d C_{t}\right)=\mathbf{N},
$$

where $\mathbf{M}=\left[\begin{array}{c}m_{1} \\ \vdots \\ m_{k}\end{array}\right]$ is $k \times m n$ and $\mathbf{N}=\left[\begin{array}{c}n_{1} \\ \vdots \\ n_{k}\end{array}\right]$ is $k \times 1$. This is a standard least-square problem which can be solved efficiently. Note that (32) is always underdetermined as the number of unknowns is $m n$ while the number of equations is no more than $k$ and $k \ll m n$. The passivity-enforced proper system is $\left(E_{p}(\right.$ or $\left.E), A, B, \tilde{C}, D\right)$, where $\tilde{C}=C+d C_{t} L^{-T}$.

3) Displacement of Purely Imaginary Eigenvalues: In this subsection, we discuss the choice of $\tilde{\omega}_{i}$. Nonetheless, the criterion of this choice is nontrivial and somewhat relies on experience. First consider the sign of the displacement. Suppose that the eigenvalue (denoted as $\lambda_{G}$ ) of the sampling $G(j \omega)$ in the frequency band on the right of $j \omega_{i}$ is positive and that on the left of $j \omega_{i}$ is negative, the sign of displacement is minus. Otherwise the sign is plus. Note that $\lambda_{G}$ is always real as $G(j \omega)$ is Hermitian.

Then consider the amount of the displacement. Without loss of generality, assume that $\lambda_{G}$ on the right of $j \omega_{i}$ is negative. Analysis shows that the amount of displacement depends on both the bandwidth $W_{B}=\omega_{i+1}-\omega_{i}$ and $\left|\lambda_{G}\right|$ (positive correlation). Therefore, the criterion for choosing $\tilde{\omega}_{i}$ is stated as below:

$$
\left|\tilde{\omega}_{i}-\omega_{i}\right|=\eta\left(\left|\lambda_{G}\right|\right) W_{B}
$$

Here the coefficient $\eta\left(\left|\lambda_{G}\right|\right)$ is a monotonically increasing function of $\left|\lambda_{G}\right|$ and its range is $(0,0.5]$.

\section{Further Discussion on D Term}

The above passivity characterization and passivity enforcement algorithms rely on the assumption that $D+D^{T}$ is nonsingular. However, this assumption is in general not satisfied for a large number of real systems. For example, $D=0$ in the MNA model of RCL circuits and Loewner-TI macromodels. Therefore, an equivalent model conversion is necessary, through which $D$ terms can be made nonsingular without changing the transfer function of the system [24]. Assume that $\kappa>0$ is not an eigenvalue of $D$ (if $D=0$, assign $\kappa=1$ ), we have $\kappa I-D$ being invertible. Thus the original system can be converted to:

$$
\begin{gathered}
E_{e q}=\left[\begin{array}{ll}
E & \\
& 0
\end{array}\right], \quad A_{e q}=\left[\begin{array}{ll}
A & \\
& (\kappa I-D)^{-1}
\end{array}\right], \\
B_{e q}=\left[\begin{array}{c}
B \\
I
\end{array}\right], \quad C_{e q}=\left[\begin{array}{ll}
C & I
\end{array}\right], \quad D_{e q}=\kappa I .
\end{gathered}
$$

It is straightforward to verify that the transfer function of the equivalent model (34) is identical to that of the original model [24]. $D_{e q}+D_{e q}^{T}(=2 \kappa I)$ is guaranteed to be nonsingular. Besides, the model conversion does not change the index of the model. 


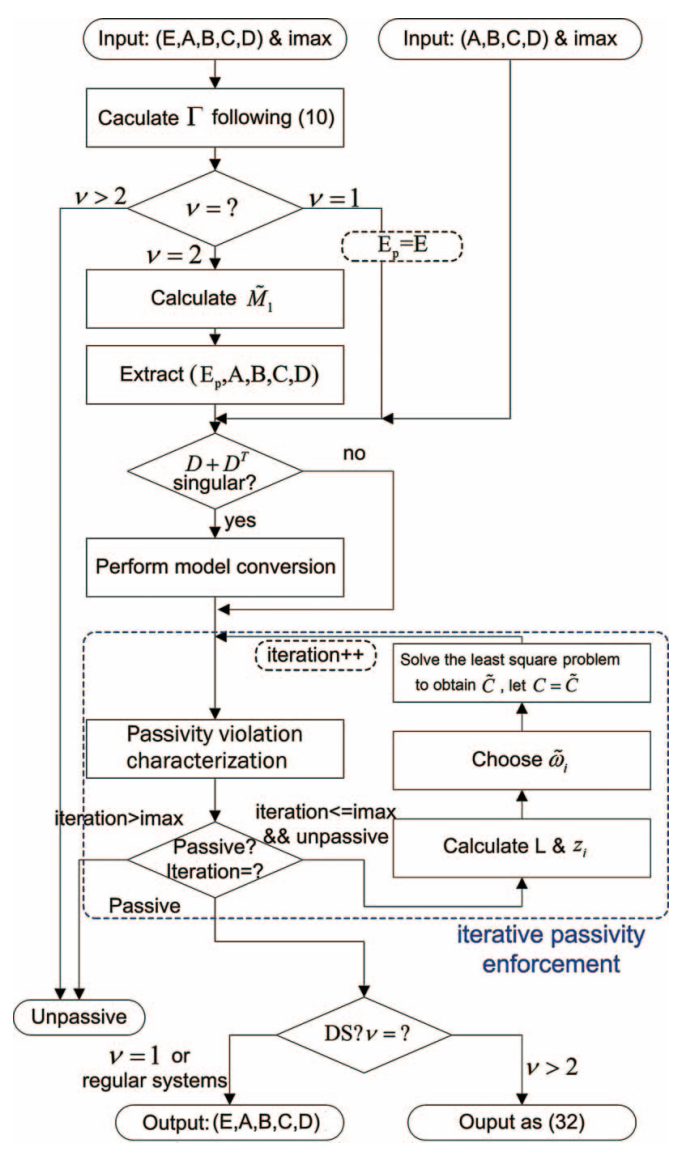

Fig. 1. DS passivity enforcement flow.

\section{Summary of Algorithm}

After the passivity enforcement of the improper (if exists) and proper parts, the system is guaranteed to be passive. The transfer function of the passive DS comprises of two parts: that of $\left(E_{p}, A, B, \tilde{C}, D\right)$ and $s \tilde{M}_{1}$. Then the system can be represented as a combination of the two parts. The new DS can be optionally reconstructed as:

$$
\begin{gathered}
E^{\prime}=\left[\begin{array}{rrr}
E_{p} & & \\
& 0 & I_{m} \\
& 0 & 0
\end{array}\right], \quad A^{\prime}=\left[\begin{array}{lll}
A_{p} & & \\
& I_{m} & \\
& & I_{m}
\end{array}\right] \\
B^{\prime}=\left[\begin{array}{c}
B \\
0 \\
\tilde{L}^{T}
\end{array}\right], \quad C^{\prime}=\left[\begin{array}{lll}
\tilde{C}-\tilde{L} & 0
\end{array}\right], \quad D^{\prime}=D,
\end{gathered}
$$

where $\tilde{M}_{1}=\tilde{L}^{T} \tilde{L}$ is the Cholesky factorization of $\tilde{M}_{1}$. One can easily check that the transfer function of this DS is identical to the sum of the proper and improper parts. Besides, $A^{\prime}$ remains nonsingular if $A$ is nonsingular and $E^{\prime}$ is index2 if $E$ is index-2 (note that the matrix block $\left[\begin{array}{cc}0 & I_{m} \\ 0 & 0\end{array}\right]$ is index-2).

Passivity enforcement of the proper part requires solving the optimization problem (32), whose efficiency is influenced by the partially empirical choice of $\omega_{i, p}$. Thus the perturbed DS should be treated as a new input and go through the passivity check procedure. If nonpassive, iterative perturbations should be performed. The flow of PEDS is summarized as Algorithm 1 (Fig. 1). In practice, the iteration number is usually no more than 5 if the passivity violation is mild (which ought to be the case when the underlying system being modeled is passive).

\section{E. Computation Complexity}

1) Calculation of $\nu$ and improper part extraction: This procedure only involves vector-matrix multiplication and vector-matrix solving, thus its computation complexity is low.

2) System decomposition via canonical projector methods: Computation complexity of this procedure is $O\left(n^{3}\right)$, which can be further speeded up by employing (sparse) LU decomposition in the spectral projector calculation of the (sparse) DS [22]. The canonical projector-based decomposition is faster and numerically more stable compared to direct calculation of Weierstrass canonical form. Moreover, this procedure can be totally avoided for most commonly encountered impulse-free DSs.

3) Passivity enforcement of the improper part: The size of the extracted improper part is $m$, where $m$ is the number of ports (small). Thus the size of the LMI is $m^{2}+1$. Computation complexity of this procedure is negligible among the flow.

4) Iterative passivity check and enforcement of the proper part: This procedure dominates the speed of the algorithm. In each iteration, an $O\left(n^{3}\right)$ generalized eigenvalue calculation is required. If the matrices are sparse, cheaper eigenvalue calculation algorithms for sparse matrices can be employed. For most cases, the passivity enforcement can be finished in no more than 5 iterations.

5) Model conversion and reconstruction: Their computation complexity is negligible.

In summary, the complexity of the algorithm is $O\left(n^{3}\right)$ and is dominated by the iterative passivity test and enforcement procedure. Therefore, PEDS can handle small or medium models or large but sparse models. Fortunately, this is not a problem in real applications as most real-world models are sparse and MOR is normally first performed on large scale models.

\section{NUMERICAL EXAMPLES}

\section{Example 1: An illustrative example}

The model studied is an artificial single-input-single-output, order-4 DS in impedance/admittance representation. The index of the DS is 2 . The system is stable with two finite poles $(-0.5000 \pm j 1.4142)$ in the open left half plane.

$$
\begin{aligned}
& E=\left[\begin{array}{rrrr}
16 & 12 & -4 & 14 \\
14 & 8 & 4 & -14 \\
-14 & 8 & -4 & 34 \\
6 & -4 & 0 & -10
\end{array}\right], A=\left[\begin{array}{rrrr}
6 & -19 & 7 & -9 \\
11 & 3 & -21 & 18 \\
25 & -9 & 35 & -16 \\
-27 & 6 & -16 & 38
\end{array}\right] \\
& B^{T}=\left[\begin{array}{llll}
-0.6 & 1 & 0.2 & -0.3
\end{array}\right], \quad C=\left[\begin{array}{llll}
3.2 & 1.4 & 2.6 & 1.4
\end{array}\right], \quad D=0.105, \\
& \tilde{C}=\left[\begin{array}{llll}
3.0876 & 1.4736 & 2.6 & 1.4
\end{array}\right] .
\end{aligned}
$$




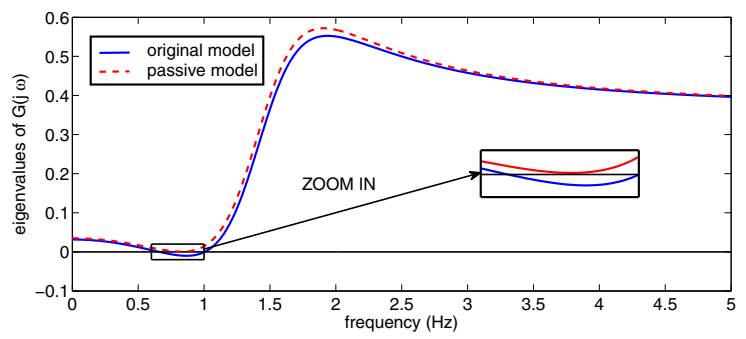

Fig. 2. Eigenvalue plot of $G(j \omega)$, for example 1

By calculating $\Gamma$, we get that the index of the DS is 2 and $M_{1}=0.2$, which is already positive definite. Then system decomposition is performed and the proper part is extracted. Passivity check demonstrates that passivity violation occurs in the frequency band $(0.66 \mathrm{~Hz}, 1.01 \mathrm{~Hz})$. Then the proper part is enforced to be passive by perturbing $C$ to $\tilde{C}$ [cf. (36)]. The eigenvalue plot of $G(j \omega)$ is shown in Fig. 2, which shows that no negative eigenvalues appear for the perturbed model. The Bode diagrams of the original and passive models almost overlap each other, which indicates that the passivity-enforced model enjoys good accuracy. The perturbation of $C$ is small as $\|\tilde{C}-C\| /\|C\|=0.0294$.

\section{Example 2: A PEEC reduced-order model}

The model used in this example is a reduced-order model of an original order-480 PEEC model, which is generated by partial element equivalent circuit modeling of a patch antenna [2]. The original model is a single-input-single-output DS with $D=0$. A order-35 reduced order model is obtained through PRIMA [15]. All poles of the reduced model are stable. An impulse response test following Section III-A shows that the model is impulse-free. The passivity violation band is from 1.15 to $1.39(\mathrm{rad} / \mathrm{s})$.

The order-35 nonpassive model is in DS form with $D+$ $D^{T}=0$, hence previous passivity enforcement algorithms are not applicable. PEDS is performed on it for passivity compensation. Since the model is impulse-free, no system decomposition is required. Passivity is achieved by perturbing $C$ to $\tilde{C}$. The eigenvalue plots of the original and passive model are shown in Fig. 3. It can be seen that passivity is enforced without significantly impacting the frequency response of the model.

\section{Example 3: A common mode filter}

The data used in this model is from TDK S-parameter data library [20]. The S-parameter data are measured from an 8port common mode filter. The S-parameter matrices are then converted to Y-parameter (admittance representation) ones. A DS model is generated from the Y-parameter matrices through Loewner-TI, which is an efficient method to build models from frequency domain sampled response, especially for systems with massive ports. The model generated is an order-167 DS with $D=0$. A simple calculation shows that all the finite poles

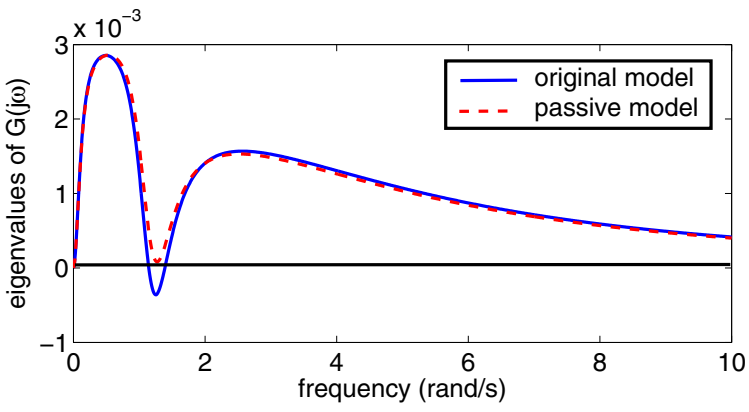

Fig. 3. Eigenvalue plot of $G(j \omega)$, for example 2

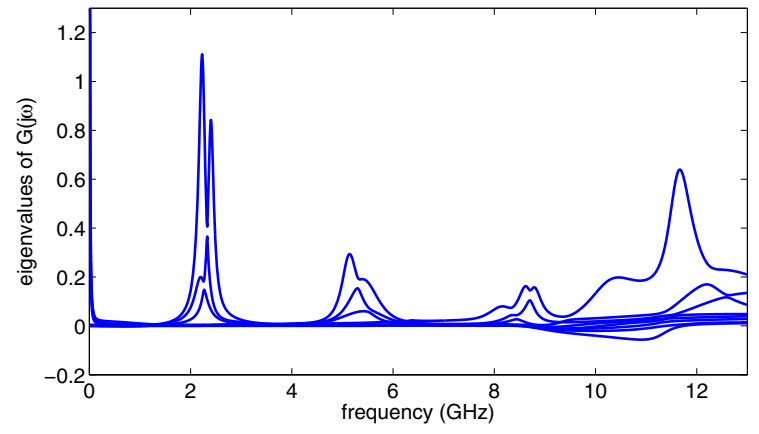

Fig. 4. Eigenvalue plot of the original model

of the generated model are stable. But nonpassivity occurs due to the modeling error. 40 purely imaginary generalized eigenvalues of the Hamiltonian-symplectic matrix pencil are detected, which implies 20 crossover points in the eigenvalue plot of $G(j \omega)$.

PEDS is performed on the generated DS model. As the DS is impulse-free, no system decomposition is required. Purely imaginary generalized eigenvalues are moved off the imaginary axis through iterative solves of the least-square problems. The $G(j \omega)$ eigenvalue plots of the original and perturbed models are shown in Figs. $4 \&$ 5. Fig. 6 shows the zoomed in portion between $8 \mathrm{GHz}$ and $11.5 \mathrm{GHz}$. The perturbed model is guaranteed passive, with little accuracy loss. We choose the port-2 to port-1 Bode diagram to show the accuracy of the perturbed model. As shown in Fig. 7, the perturbed model is accurate in most frequency bands.

\section{CONCLUSION}

The passivity enforcement algorithm for DSs based on perturbation of Hamiltonian-symplectic matrix pencil, PEDS, has been proposed. PEDS is the first algorithm that handles more general DS models and DS/RS models with singular $D+D^{T}$. PEDS can minimize the error of the passivity enforced models through a coordinate transformation method. The perturbations of the improper part and proper part of the DS are finally reduced to a standard LMI "mincx" problem and a standard least-square problem. Numerical examples have demonstrated that PEDS is efficient, fast and numerically stable for DSs with mild passivity violations. 


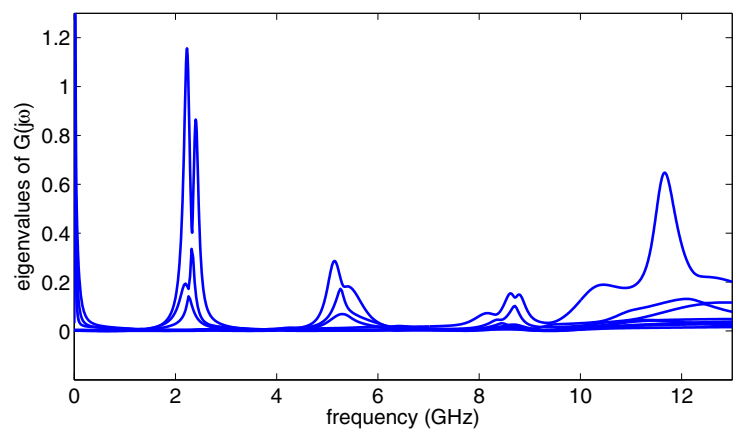

Fig. 5. Eigenvalue plot of the passive model

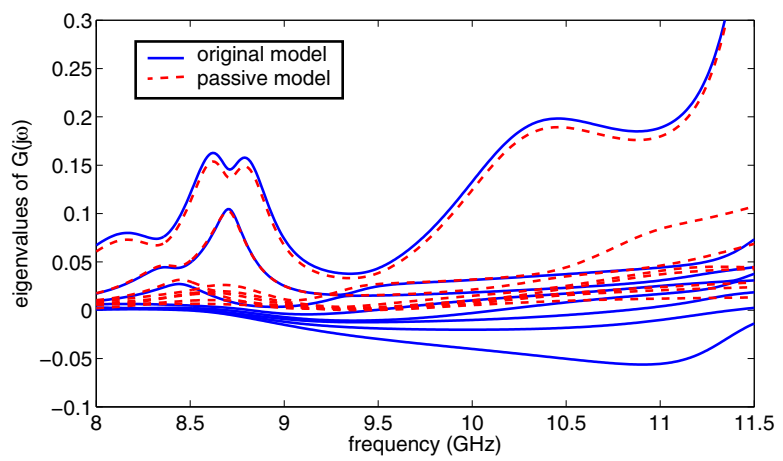

Fig. 6. Eigenvalue plot of the original and passive models: zoom in in the frequency band between $8 \mathrm{GHz}$ and $11.5 \mathrm{GHz}$

\section{REFERENCES}

[1] S. Boyd, V. Balakrishnan, and P. Kabamba. A bisection method for computing the $H_{\infty}$ norm of a transfer matrix and related problems. Mathematics of Control, Signals, and Systems (MCSS), 2(3):207-219, 1989.

[2] Y. Chahlaoui and P. Van Dooren. A collection of benchmark examples for model reduction of linear time invariant dynamical systems. SLICOT working note, 2002.

[3] L. Dai. Singular control systems. Berlin and New York, Springer-Verlag, 1989.

[4] P. Feldmann and R. Freund. Reduced-order modeling of large linear subcircuits via a block Lanczos algorithm. In Proc. IEEE/ACM Design Automation Conference, page 479, 1995.

[5] R. Freund and F. Jarre. An extension of the positive real lemma to descriptor systems. Optimization methods and software, 19(1):69-87, 2004.

[6] S. Grivet-Talocia. Passivity enforcement via perturbation of Hamiltonian matrices. IEEE Transactions on Circuits and Systems I Regular Papers, 51(9):1755-1769, 2004.

[7] B. Gustavsen and A. Semlyen. Enforcing passivity for admittance matrices approximated by rational functions. IEEE Transactions on Power Systems, 16(1):97-104, 2001.

[8] B. Gustavsen, A. Semlyen, and T. EFI. Rational approximation of frequency domain responses by vector fitting. IEEE Transactions on Power Delivery, 14(3):1052-1061, 1999.

[9] C. Ho, A. Ruehli, and P. Brennan. The modified nodal approach to network analysis. IEEE Transactions on Circuits and Systems, 22(6):504 509, 1975.

[10] E. Kuh and R. Rohrer. Theory of linear active networks. Holden-day, 1967.

[11] S. Lefteriu and A. C. Antoulas. A New Approach to Modeling Multiport Systems From Frequency-Domain Data. IEEE Transaction on ComputerAided Design of Integrated Circuits and Systems, 29(1):14-27, 2010.

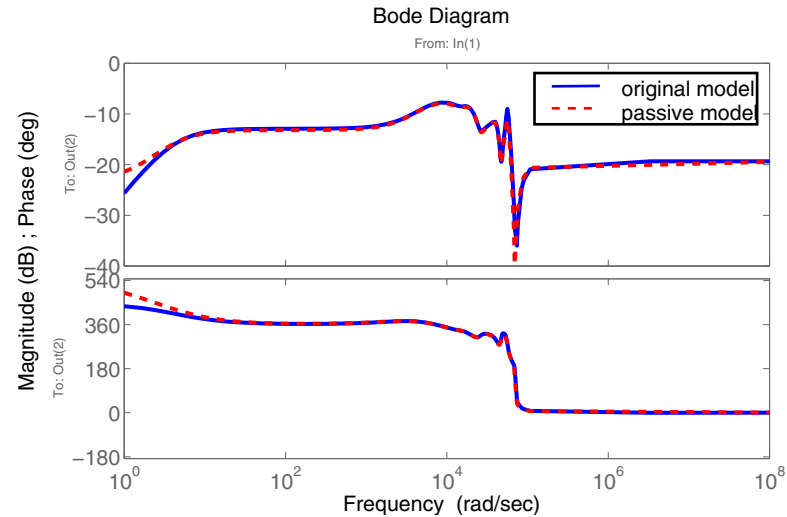

Fig. 7. Bode diagram of the original and the passive models: port- 2 to port- 1

[12] J. Li, F. Wang, and J. White. An efficient Lyapunov equation-based approach for generating reduced-order models of interconnect. In Proc. IEEE/ACM conference on Design automation, pages 1-6, 1999.

[13] Y. Liu and N. Wong. Fast sweeping methods for checking passivity of descriptor systems. In IEEE Asia Pacific Conference on Circuits and Systems, pages 1794-1797, 2008.

[14] R. Marz. Canonical projectors for linear differential algebraic equations. Computers and Mathematics with Applications, 31(4-5):121-135, 1996.

[15] A. Odabasioglu, M. Celik, and L. Pileggi. PRIMA: Passive and reducedorder interconnect macromodeling algorithm. IEEE Transaction on Computer-Aided Design of Integrated Circuits and Systems, 17(8):645654, 1998.

[16] J. Phillips, L. Daniel, and L. Silveira. Guaranteed passive balancing transformations for model order reduction. In In Proc. IEEE/ACM Design Automation Conference, pages 52-57, 2002.

[17] A. Ruehli, G. Antonini, J. Esch, J. Ekman, A. Mayo, and A. Orlandi. Nonorthogonal PEEC formulation for time-and frequency-domain EM and circuit modeling. IEEE Transactions on Electromagnetic Compatibility, 45(2):167-176, 2003.

[18] D. Saraswat, R. Achar, and M. Nakhla. Global passivity enforcement algorithm for macromodels of interconnect subnetworks characterized by tabulated data. IEEE Transactions on Very Large Scale Integration (VLSI) Systems, 13(7):819-832, 2005.

[19] T. Stykel. Gramian-based model reduction for descriptor systems. Mathematics of Control, Signals, and Systems (MCSS), 16(4):297-319, 2004.

[20] TDK. TDK S-parameter data library, 2009. http://www.tdk.com/tvcl_ sparam.php.

[21] Y. Wang, C. Lei, G. Pang, and N. Wong. MFTI: matrix-format tangential interpolation for modeling multi-port systems. In Proceedings of the 47th Design Automation Conference, pages 683-686, 2010.

[22] N. Wong. An efficient passivity test for descriptor systems via canonical projector techniques. In Proc. IEEE/ACM Design Automation Conference, pages 957-962, 2009.

[23] L. Zhang, J. Lam, and S. Xu. On positive realness of descriptor systems. IEEE Transactions on Circuits and Systems I: Fundamental Theory and Applications, 49(3):401-407, 2002.

[24] Z. Zhang, C. Lei, and N. Wong. GHM: a generalized Hamiltonian method for passivity test of impedance/admittance descriptor systems. In Proc. IEEE/ACM International Conference on Computer-Aided Design, pages 767-773, 2009.

[25] Z. Zhang and N. Wong. An Efficient Projector-Based Passivity Test for Descriptor Systems. IEEE Transaction on Computer-Aided Design of Integrated Circuits and Systems, 29(8):1203-1214, 2010. 